\title{
SOME LOCALLY CONVEX SPACES OF CONTINUOUS VECTOR-VALUED FUNCTIONS OVER A COMPLETELY REGULAR SPACE AND THEIR DUALS
}

\author{
BY
}

\section{A. KATSARAS}

ABSTRACT. The strict, superstrict and the $\beta_{F}$ topologies are defined on a space $A$ of continuous functions from a completely regular space into a Banach space $E$. Properties of these topologies are discussed and the corresponding dual spaces are identified with certain spaces of operator-valued measures. In case $E$ is a Banach lattice, $A$ becomes a lattice under the pointwise ordering and the strict and superstrict duals of $A$ coincide with the spaces of all $\boldsymbol{\tau}$-additive and all $\sigma$-additive functionals on $A$ respectively.

Introduction. The Riesz representation theorem says that any continuous linear functional $F$ on the space of continuous real functions on a compact Hausdorff space $X$ with the uniform topology must have the form $F(f)=\int_{X} f d m$ for some bounded regular Borel measure on $X$. This representation was extended later to other spaces, first in case $X$ is locally compact and later by Aleksandrov [1] for continuous linear functionals on the space $C^{b}(X)$ of all bounded continuous real functions on a completely regular space. The representation was given by means of integrals with respect to members of the space $M(X)$ of all bounded, finitely-additive, regular with respect to zero sets, measures on the algebra generated by the zero sets. The $\sigma$-additive, $\tau$-additive and tight linear functionals correspond to the $\sigma$-additive, $\tau$-additive and tight members of $M(X)$ respectively (see Varadarajan [24]). Buck [4], for the locally compact case, and Sentilles [23], for the completely regular case, have defined the strict topologies on $C^{b}(X)$ which yield as dual spaces certain subspaces of $M(X)$. Several others like Hewitt [10], Bogdanowich [2], Wells [25], the author [12] and others have considered the problem of representation of linear functionals on spaces of continuous scalarvalued or vector-valued functions. In this paper we define certain locally convex topologies on spaces of continuous vector-valued functions on a completely regular space. We study some of the properties of these topologies and represent their duals with operator-valued measures on certain $\sigma$-algebras of subsets of $X$. The

Received by the editors December 20, 1972 and, in revised form, April 15, 1975. AMS (MOS) subject classifications (1970). Primary 46E40; Secondary 46A05, 46A40.

Key words and phrases. Locally convex spaces, strict topology, mixed topology, operator-valued measures, $\sigma$-additive functionals, $\tau$-additive functionals. 
integration process employed is a generalization of the process of Aleksandrov to the vector case. It is one of the many integration processes defined by McShane [17].

1. Definition and notation. Throughout this paper $X$ will denote a completely regular Hausdorff space and $Y$ will be a Hausdorff compactification of $X$. We will denote by $B$ the algebra of continuous real-valued functions $f$ on $X$ which have continuous extensions $\hat{f}$ to all of $Y$. Let $E$ be a Banach space over the real field. We will denote by $A$ the space of all continuous functions $f$ from $X$ into $E$ which have continuous extensions $\hat{f}$ to all of $Y$. Let $C=\{\hat{f}: f \in A\}$. If $f \in A$ and $g \in B$, the function $g f$ is defined on $X$ by $(g f)(x)=g(x) f(x)$. For $s \in E$ we will denote also by $s$ the element of $A$ whose value at every $x$ is equal to $s$.

We will consider on $A$ various locally convex topologies.

(a) The uniform topology $\sigma$ generated by the norm

$$
f \rightarrow\|f\|=\sup \{\|f(x)\|: x \in X\} .
$$

(b) The topology $k$ generated by the family seminorms $p_{K}, K$ compact in $X$, where

$$
p_{K}(f)=\|f\|_{K}=\sup \{\|f(x)\|: x \in K\} .
$$

(c) The topology $\pi$ generated by the family of seminorms $P_{x}, x \in X$, where

$$
p_{x}(f)=\|f(x)\| \text {. }
$$

Clearly all topologies $\pi, k, \sigma$ are Hausdorff and $\pi \leqslant k \leqslant \sigma$. Finally, if $\tau$ is a linear topology on $A$, then $(A, \tau)^{\prime}$ denotes the topological dual of $(A, \tau)$.

2. The strict and superstrict topologies on $A$. Buck [4] defined the strict topology on the space of bounded continuous functions on a locally compact Hausdorff space. This topology has been studied later by several other authors. Recently Sentilles [23] defined the strict and superstrict topologies on the family of all bounded, continuous, real-valued functions on a completely regular Hausdorff space. In this section we will define the strict and superstrict topologies on the space $A$ defined in $\S 1$. Our approach will be analogous to that of Sentilles. Several of our theorems will be generalizations of his results.

A subset $Z$ of $Y$ is called a zero set if $Z=f^{-1}\{0\}$ for some continuous real function $f$ on $Y$. We will denote by $\Omega\left(\Omega_{1}\right)$ the class of all closed (zero) subsets of $Y$ which are disjoint from $X$. For a $Q$ in $\Omega$, let $B_{Q}=\{f \in B: \hat{f}=0$ on $Q$ \}. It is not hard to see that $B_{Q}$ is a Banach algebra (under the uniform norm) with an approximate identy of norm $\leqslant 1$.

Let $Q \in \Omega$. We will denote by $\beta_{Q}$ the locally convex topology on $A$ generated by the family of seminorms $f \rightarrow\|g f\|, g \in B_{Q}$. The space $(A, \sigma)$ is a Banach space and a $B_{Q}$-module since $g f \in A$ for every $g \in B_{Q}$ and every $f \in A$. The topology $\beta_{Q}$ is the strict topology on $A$ as defined by Sentilles [20]. Hence 
$\beta_{Q}$ is the finest locally convex topology on $A$ which agrees with $\beta_{Q}$ on norm bounded subsets of $A$ by Sentilles [21, Theorem 2.2]. A convex balanced absorbent set $W$ in $A$ is a $\beta_{Q}$-neighborhood of zero iff given $r>0$ there exists a $\beta_{Q}$ neighborhood $V$ of zero such that $U_{r} \cap V \subset W$, where $U_{r}=\{f \in A:\|f\| \leqslant r\}$.

The strict topology $\beta=\beta(A)$ on $A$ is defined to be the inductive limit of the topologies $\beta_{Q}, Q \in \Omega$. The superstrict topology $\beta_{1}$ is the inductive limit of the topologies $\beta_{Z}, Z \in \Omega_{1}$. By definition of the inductive limit topology (see Schaefer [19, p. 57]), a convex balanced absorbent subset $W$ of $A$ is a $\beta\left(\beta_{1}\right)$ neighborhood of zero iff $W$ is a $\beta_{Q}$-neighborhood of zero for each $Q \in \Omega\left(Q \in \Omega_{1}\right)$.

THEOREM 2.1. $k \leqslant \beta \leqslant \beta_{1} \leqslant \sigma$.

Proof. It is clear that $\beta \leqslant \beta_{1} \leqslant \sigma$. To prove that $k \leqslant \beta$ consider an arbitrary compact set $K$ in $X$. We want to show that the set $W=\left\{f \in A:\|f\|_{K} \leqslant 1\right\}$ is a $\beta$-neighborhood of zero. Since $W$ is convex balanced and absorbent it suffices to show that $W$ is a $\beta_{Q}$-neighborhood of zero for every $Q$ in $\Omega$. So, let $Q \in \Omega$. Since $K$ is compact, there exists $g \in B$ such that $g=1$ on $K$ and $\hat{g}=0$ on $Q$. Then $g \in B_{Q}$ and $V=\{f \in A:\|g f\| \leqslant 1\} \subset W$. Since $V$ is a $\beta_{Q}$-neighborhood of zero the result follows.

Since, for each $Q \in \Omega, \beta_{Q}$ is the finest locally convex topology on $A$ which agrees with $\beta_{Q}$ on norm bounded subsets of $A$, it follows that $\beta\left(\beta_{1}\right)$ is the finest locally convex topology $\tau$ on $A$ which agrees with $\beta\left(\beta_{1}\right)$ on norm bounded sets.

Lemma 2.2. Let $\pi$ be a locally convex topology on $A$ such that $\pi \leqslant \tau \leqslant \sigma$ and such that $(A, \tau)^{\prime}=H$ is a norm closed subspace of $A^{\prime}=(A, \sigma)^{\prime}$. Then $\tau$ and $\sigma$ have the same bounded sets.

Proof. Every $\sigma$-bounded set is obviously $\tau$-bounded. On the other hand, suppose that $G$ is a $\tau$-bounded subset of $A$. Then $G$ is $\sigma(A, H)$ bounded. By our hypothesis $H$ is a Banach space under the norm

$$
\phi \rightarrow\|\phi\|=\sup \{|\phi(f)|: f \in A,\|f\| \leqslant 1\} .
$$

Each $f \in A$ defines a bounded linear functional $T_{f}$ on $H$ by $T_{f}(\phi)=\phi(f)$. Since $G$ is $\sigma(A, H)$ bounded, we have $\sup \left\{\left|T_{f}(\phi)\right|: f \in G\right\}<\infty$ for each $\phi \in H$. By the principle of uniform boundedness there exists $K>0$ such that $\sup \left\{\left\|T_{f}\right\|\right.$ : $f \in G\} \leqslant K$. Let now $f \in G$ and $x \in X$. By the Hahn-Banach theorem there is a $T$ in $E^{\prime},\|T\| \leqslant 1, T(f(x))=\|f(x)\|$. Define $\pi_{x}: A \rightarrow R, \pi_{x}(g)=T(g(x))$. Then $\pi_{x}$ is in $H$ since $\pi_{x} \in(A, \pi)^{\prime} \subset H$. Moreover $\left\|\pi_{x}\right\| \leqslant 1$. Thus $\|f(x)\|=\pi_{x}(f)=$ $T_{f}\left(\pi_{x}\right) \leqslant K$. It follows that $\sup \{\|f\|: f \in G\} \leqslant K$ which completes the proof.

THEOREM 2.3. Let $\tau$ be as in Lemma 2.2. The following are equivalent:

(1) $\tau=\sigma$.

(2) $\tau$ is normable. 
(3) $\tau$ is metrizable.

(4) $\tau$ is bornological.

(5) $\tau$ is barrelled.

Proof. It is clear that (1) implies (2), (2) implies (3), and (3) implies (4). To prove that (4) implies (5), we first observe that the set $U_{1}=\{f \in A:\|f\| \leqslant 1\}$ is convex balanced and absorbs every norm (and hence every $\tau$ ) bounded set. By (4) $U_{1}$ is a $\tau$-neighborhood of zero. It follows that $\tau=\sigma$. Since $(A, \sigma)$ is a Banach space, (5) follows. Finally to prove that (5) implies (1) we observe that the set $U_{1}$ is $\pi$-closed and hence $\tau$-closed. Since $U_{1}$ is also convex balanced and absorbent it follows that $U_{1}$ is a $\tau$-neighborhood of zero and hence $\tau=\sigma$.

THEOREM 2.4. The duals of the spaces $(A, \beta)$ and $\left(A, \beta_{1}\right)$ are norm closed subspaces of the dual of $(A, \sigma)$.

Proof. Let $\left\{T_{n}\right\}$ be a sequence in $(A, \beta)^{\prime} . T \in A^{\prime}$, such that $\left\|T_{n}-T\right\|$ $\rightarrow 0$. Let $W=\{f \in A:|T(f)| \leqslant 1\}$. We need to show that $W$ is a $\beta$-neighborhood of zero. Since $W$ is convex balanced and absorbent it suffices to show that given $r>0$ there exists a $\beta$-neighborhood $V$ of zero such that $V \cap\{f \in A:\|f\| \leqslant$ $r\} \subset W$. So, let $r>0$. Choose $n$ so that $\left\|T_{n}-T\right\|<1 /(2 r)$. Let $V=\{f \in A$ : $\left.\left|T_{n}(f)\right|<1 / 2\right\}$. Then $V$ is a $\beta$-neighborhood of zero and $V \cap\{f \in A:\|f\| \leqslant r\}$ $\subset W$. This proves the result for $\beta$. The proof for $\beta_{1}$ is similar.

Corollary 2.5. (a) Theorem 2.3 holds for $\tau=\beta$ or $\beta_{1}$.

(b) $\beta, \beta_{1}$ and $\sigma$ have the same bounded sets.

THEOREM 2.6. The topologies $\beta$ and $\sigma$ coincide iff $X$ is compact.

Proof. Clearly $\beta=\sigma$ when $X$ is compact. On the other hand assume that $X$ is not compact. Then $Y \neq X$. Let $x \in Y-X, Q=\{x\}$. Let $g \in B_{Q}$ and set $V=\{f \in A:\|g f\| \leqslant 1\}$. Choose $s \in E,\|s\|=2$ and set $F=\{y \in Y:|\hat{g}(y)|$ $\geqslant 1 / 2\}$. There exists $h \in B, 0 \leqslant h \leqslant 1$, such that $\hat{h}=0$ on $F$ and $\hat{h}(x)=1$. Then the function $f=h s$ is in $V$ but not in $U_{1}=\{f \in A:\|f\| \leqslant 1\}$. Hence $U_{1}$ does not contain $V$. It follows that $U_{1}$ is not a $\beta_{Q}$-neighborhood of zero and hence $\beta \neq \sigma$.

3. The topology $\beta_{F}$. Let $F$ be a collection of compact subsets of $X$ satisfying the following two conditions:

(1) $\bigcup F=X$.

(2) $F$ is directed, i.e. given $G_{1}, G_{2}$ in $F$ there exists $G$ in $F$ containing both $G_{1}$ and $G_{2}$. We denote by $\tau_{F}$ the locally convex topology on $A$ generated by the family of seminorms $\left\{\|\cdot\|_{G}: G \in F\right\}$, where $\|f\|_{G}=\sup \{\|f(x)\|: x \in G\}$. The topology $\beta_{F}=\beta_{F}(A)$ is defined to be the mixed topology $\gamma\left[\sigma, \tau_{F}\right]$ as defined by Wiweger [26]. By Wiweger we have $\tau_{F} \leqslant \beta_{F} \leqslant \sigma$ and that $\beta_{F}$ is the finest locally 
convex topology on $A$ which agrees with $\tau_{F}$ on each norm bounded subset of $A$ (see Wiweger [26, 2.2.2.]).

LEMMA 3.1. Let $G_{1}, \ldots, G_{n}$ be in $F, \epsilon>0$ and $f$ in $A$. Then there exist $g, h$ in $A$ such that $f=g+h, h=0$ on each $G_{i}$, and $\|g\| \leqslant \epsilon+\max \left\{\|f\|_{G_{i}}\right.$ : $1 \leqslant i \leqslant n\}$.

Proof. Let $G=\bigcup G_{i}$ and set $d=\epsilon+\|f\|_{G}$. Then $G \subset V=\{x \in Y$ : $|\hat{f}(x)|<d\}$. Since $G$ is compact and $V$ open there exists $h_{0} \in B, 0 \leqslant h_{0} \leqslant 1$, $\hat{h}_{0}=1$ on $G, \hat{h}_{0}=0$ on $Y-V$. Set $g=f h_{0}$ and $h=f\left(1-h_{0}\right)$. Then $g$ and $h$ satisfy the requirements.

Corollary 3.2. The sets of the form $\bigcap_{i=1}^{\infty}\left\{f \in A:\|f\|_{G_{i}} \leqslant a_{i}\right\}$, where $0<a_{i} \rightarrow \infty$ and $G_{i} \in F$, constitute $a \beta_{F}$-neighborhood base at zero.

Proof. It follows from the preceding lemma and from Wiweger's Theorem 3.1.1.

We will next give an alternative description of $\beta_{F}$. Denote by $B_{0}(F)$ the collection of all bounded real-valued functions $f$ on $X$ with the property that given $\epsilon>0$ there exists $G$ in $F$ such that $\{x \in X:|f(x)| \geqslant \epsilon\} \subset G$. We define on $A$ the locally convex topology $\tau(F)$ generated by the family of seminorms $P_{g}, g \in$ $B_{0}(F)$, where

$$
P_{g}(f)=\|g f\|=\sup \{\|g(x) f(x)\|: x \in X\} .
$$

LEMMA 3.3. The topologies $\tau(F)$ and $\tau_{F}$ coincide on each $\sigma$-bounded subset of the space $A$.

Proof. Let $r>0$ and set $U_{r}=\{f \in A:\|f\| \leqslant r\}$. Suppose that $V$ is a subset of $U_{r}$ which is closed with respect to the relative topology of $\tau(F)$ on $U_{r}$. We will show that $V$ is closed with respect to the $\tau_{F}$ relative topology on $U_{r}$. Indeed, let $f \in U_{r}$ be in the $\tau_{F}$ closure of $V$. Let $g \in B_{0}(F)$. Given $\epsilon>0$ there exists $G$ in $F$ such that $|g(x)|<\epsilon /(2 r)$ if $x$ is not in $G$. Choose $h$ in $V$ with $\|f-h\|_{G}<\epsilon /\|g\|$. Then $\|g(f-h)\| \leqslant \epsilon$. This proves that $f$ is in the $\tau(F)$ closure of $V$ and hence in $V$. It follows that $\left.\tau(F)\right|_{U_{r}} \leqslant\left.\tau_{F}\right|_{U_{r}}$. On the other hand $\tau_{F} \leqslant \tau(F)$ because the characteristic function of any set in $F$ belongs to $B_{0}(F)$. We conclude that $\tau(F)$ and $\tau_{F}$ agree on $U_{r}$. The result follows.

\section{THEOREM 3.4. The topologies $\tau(F)$ and $\beta_{F}$ coincide.}

Proof. Since $\beta_{F}$ is the finest locally convex topology on $A$ which agrees with $\tau_{F}$ on norm bounded subsets of $A$, we have $\tau(F) \leqslant \beta_{F}$ by 3.3. On the other hand, let $W=\bigcap_{i=1}^{\infty}\left\{f \in A:\|f\|_{G_{i}} \leqslant a_{i}\right\}$ where $0<a_{i} \rightarrow \infty$ and $G_{i} \in F$. Define $g=\sup _{i} a_{i}^{-1} \chi_{G_{i}}\left(\chi_{G_{i}}\right.$ is the characteristic function of $\left.G_{i}\right)$. Then $g \in$ $B_{0}(F)$. Moreover, $\{h \in A:\|g h\| \leqslant 1\} \subset W$. Hence $W$ is a $\tau(F)$-neighborhood of zero. Now Corollary 3.2 completes the proof. 
THEOREM 3.5. The topology $\beta_{F}$ is weaker than $\beta$.

PROOF. Let $W$ be a convex balanced absorbent $\beta_{F}$-neighborhood of zero. Let $Q \in \Omega$ and $r>0$. Since $\beta_{F}$ coincides with $\tau_{F}$ on $U_{r}$ there exist $G$ in $F$ and $\delta>0$ such that $0=\left\{f \in A:\|f\|_{G} \leqslant \delta\right\} \cap U_{r} \subset W$. Choose $g \in B_{Q}$, $0 \leqslant g \leqslant 1, g=1$ on $G$. Then $V \cap U_{r} \subset W$, where $V=\{f \in A:\|g f\| \leqslant \delta\}$. This proves that $W$ is a $\beta_{Q}$-neighborhood of zero for each $Q$ in $\Omega$. The theorem is proved.

We will next identify the space $\left(A, \beta_{F}\right)^{\prime}$. Let $L_{F}(A)$ denote the collection of all linear functionals $T$ in $A^{\prime}$ such that $T\left(f_{\alpha}\right) \rightarrow 0$ whenever $\left\{f_{\alpha}\right\}$ is a net in $U_{1}$ that converges to zero uniformly on each $G \in F$. We omit the proof of the following easily established

LEMMA 3.6. $L_{F}(A)$ is a norm closed subspace of $A^{\prime}$.

LEMma 3.7. Let $\phi \in(A, \sigma)^{\prime}$. Then $\phi$ is in $L_{F}(A)$ iff for each $\epsilon>0$ there exist $G$ in $F$ and $\delta>0$ such that $|\phi(f)| \leqslant \epsilon$ for all $f \in A$ with $\|f\| \leqslant 1$ and $\|f\|_{G} \leqslant \delta$.

Proof. The necessity of this condition is clear. To prove the sufficiency assume, by way of contradiction, that there exists $\epsilon>0$ such that for each $G \in F$ and each $\delta>0$ there exists $f=f_{(G, \delta)}$ in $A$, with $\|f\| \leqslant 1$ and $\|f\|_{G} \leqslant \delta$, such that $|\phi(f)|>\epsilon$. For $\alpha_{1}=\left(G_{1}, \delta_{1}\right), \alpha_{2}=\left(G_{2}, \delta_{2}\right)$ we write $\alpha_{1} \geqslant \alpha_{2}$ iff $G_{1} \supset$ $G_{2}$ and $\delta_{1} \leqslant \delta_{2}$. In that way we get a net $\left\{f_{\alpha}\right\}$ in $U_{1}$. Moreover, $f_{\alpha} \rightarrow 0$ uniformly on each $G \in F$. Since $\left|\phi\left(f_{\alpha}\right)\right|>\epsilon$ for all $\alpha$ we arrive at a contradiction.

THEOREM 3.8. The topological dual of the space $\left(A, \beta_{F}\right)$ is the space $L_{F}(A)$.

Proof. Let $\phi \in L_{F}(A)$. Set $W=\{f \in A:|\phi(f)| \leqslant 1\}$. If $r>0$, there exist $G \in F$ and $\delta>0$ such that $|\phi(f)| \leqslant 1 / r$ whenever $\|f\| \leqslant 1,\|f\|_{G} \leqslant \delta$. Let $V=\left\{f \in A:\|f\|_{G} \leqslant \delta r\right\}$. Then $V$ is a $\tau_{F}$-neighborhood of zero and $V \cap U_{r} \subset W$. This shows that $W$ is a $\beta_{F}$-neighborhood of zero in view of the fact that $\beta_{F}$ is the finest locally convex topology on $A$ which agrees with $\tau_{F}$ on norm bounded subsets of $A$. It follows that $\phi$ is $\beta_{F}$-continuous. Conversely, assume that $\phi$ is in $\left(A, \beta_{F}\right)^{\prime}$ and let $\epsilon>0$. There exist $G$ in $F$ and $\delta>0$ such that

$$
\left\{f \in A:\|f\|_{G} \leqslant \delta\right\} \cap U_{1 / \epsilon} \subset\{f \in A:|\phi(f)| \leqslant 1\} .
$$

Thus $|\phi(f)| \leqslant \epsilon$ whenever $f \in U_{1}$ and $\|f\|_{G} \leqslant \delta \epsilon$. By Lemma 3.7, $\phi \in L_{F}(A)$.

COROLlary 3.9. (a) $\beta_{F}$ and $\sigma$ have the same bounded sets.

(b) Theorem 2.3 holds if we replace $\tau$ with $\beta_{F}$.

4. The dual spaces of $(A, \beta),\left(A, \beta_{1}\right)$, and $\left(A, \beta_{F}\right)$. In this section we will represent the dual spaces of $(A, \beta),\left(A, \beta_{1}\right)$ and $\left(A, \beta_{F}\right)$ by means of integrals with respect to operator-valued measures. We will denote by $\operatorname{Bo}(X)$ and $\operatorname{Bo}(Y)$ 
the $\sigma$-algebras of Borel subsets of $X$ and $Y$ respectively.

The $\sigma$-algebra of Baire subsets of $Y$ will be denoted by $\operatorname{Ba}(Y)$, while the $\sigma$-algebra, of subsets of $X$, generated by the $B$-zero sets will be denoted by $\mathrm{Ba}\left(Z_{B}\right)$ (a subset $Z$ of $X$ is called a $B$-zero set if $Z=f^{-1}\{0\}$ for some $f \in B$ ).

Let $\Sigma$ be a $\sigma$-algebra of sets and let $\Sigma_{1} \subset \Sigma$. A bounded, countably-additive, real-valued, measure $m$ on $\Sigma$ is called regular with respect to $\Sigma_{1}$ if for every $G \in \Sigma$ and every $\epsilon>0$ there exists $G_{1} \in \Sigma_{1}$, contained in $G$, such that $|m(H)|<\epsilon$ for every $H \in \Sigma$ which is contained in $G-G_{1}$.

We denote by $M_{\sigma}(B)$ the space of all bounded, real-valued, countably-additive, regular with respect to the family of all $B$-zero sets, measures on $\operatorname{Ba}\left(Z_{B}\right)$. The space of all bounded, real-valued countably-additive, regular with respect to the family of zero sets in $Y$, measures on $\mathrm{Ba}(Y)$ will be denoted by $M_{\sigma}(Y)$. A regular Borel measure on $\mathrm{Bo}(X)(\mathrm{Bo}(Y))$ is a bounded, countably-additive, realvalued, measure on $\mathrm{Bo}(X)(\mathrm{Bo}(Y))$ which is regular with respect to the closed sets in $X(Y)$. A regular Borel measure $m$ on $\operatorname{Bo}(X)$ is called $\tau$-additive if $|m|\left(F_{\alpha}\right)$ $\rightarrow 0$ for each net $\left\{F_{\alpha}\right\}$ of closed sets in $X$ which decreases to the empty set. In the case of $Y$, every regular Borel measure is $\tau$-additive (see [23]).

Note. A regular Borel measure $m$ on $\operatorname{Bo}(X)$ is $\tau$-additive iff $|m|\left(Z_{\alpha}\right) \rightarrow 0$ for every net $\left\{Z_{\alpha}\right\}$ of $B$-zero sets which decreases to the empty set. Indeed, assume that the condition is satisfied and let $\left\{G_{\alpha}\right\}$ be a net of closed sets decreasing to the empty set. Since the zero sets in $Y$ form a base for the closed sets, it follows that the family of $B$-zero sets forms a base for the closed sets in $X$. Thus each $G_{\alpha}$ is an intersection of $B$-zero sets. Let

$$
D=\left\{Z \subset X: Z \text { a } B \text {-zero set, } Z \supset G_{\alpha} \text { for some } \alpha\right\} .
$$

Then $D$ is directed (by inclusion) downwards to the empty set. By hypothesis, given $\epsilon>0$, there exists $Z \in D$ with $|m|(Z)<\epsilon$. Let $\alpha_{0}$ be such that $G_{\alpha_{0}} \subset Z$. Now for each $\alpha \geqslant \alpha_{0}$ we have $|m|\left(G_{\alpha}\right) \leqslant|m|(Z)<\epsilon$ which proves that $|m|\left(G_{\alpha}\right) \rightarrow 0$.

We will denote by $M_{\tau}(X)$ and $M_{\tau}(Y)$, respectively, the spaces of all $\tau$-additive regular Borel measures on $X$ and $Y$.

Let $M_{\sigma}\left(\mathrm{Ba}\left(Z_{B}\right), E^{\prime}\right)$ denote the set of all functions $m: \mathrm{Ba}\left(Z_{B}\right) \rightarrow E^{\prime}$ with the following two properties:

(1) For each $s \in E$, the function $m s: \mathrm{Ba}\left(Z_{B}\right) \rightarrow R,(m s)(F)=m(F) s$, is in $M_{\sigma}(B)$.

(2) $|m|(X)<\infty$, where $|m|$ is defined on $\mathrm{Ba}\left(Z_{B}\right)$ by $|m|(G)=$ $\sup \left|\Sigma m\left(F_{i}\right) s_{i}\right|$, the supremum being taken over all finite partitions $\left\{F_{i}\right\}$ of $G$ into sets in $\mathrm{Ba}\left(Z_{B}\right)$ (we will refer to such a partition as a $\mathrm{Ba}\left(Z_{B}\right)$-partition) and all finite collections $\left\{s_{i}\right\} \subset E$ with $\left\|s_{i}\right\| \leqslant 1$. We define $M_{\tau}\left(\operatorname{Bo}(X), E^{\prime}\right)$ as we did $M_{\sigma}\left(\mathrm{Ba}\left(Z_{B}\right), E^{\prime}\right)$ by replacing $\mathrm{Ba}\left(Z_{B}\right)$ with $\mathrm{Bo}(X)$ and $M_{\sigma}(B)$ with $M_{\tau}(X)$. The 
spaces $M_{\sigma}\left(\mathrm{Ba}(Y), E^{\prime}\right)$ and $M_{\tau}\left(\mathrm{Bo}(Y), E^{\prime}\right)$ are defined analogously. For $m$ in any one of the above spaces, we define its norm $\|m\|$ by $\|m\|=|m|(X)$ or $|m|(Y)$ (depending on the $\sigma$-algebra on which $m$ is defined).

THEOREM 4.1. If $m \in M_{\sigma}\left(\mathrm{Ba}\left(Z_{B}\right), E^{\prime}\right)$, then $|m| \in M_{\sigma}(B)$.

Proof. It is easy to see that $|m|$ is a bounded, monotone, finitely-additive, set function on $\mathrm{Ba}\left(Z_{B}\right)$. To prove the regularity, consider a $G \in \mathrm{Ba}\left(Z_{B}\right)$ and let $\epsilon>0$ be given. By the definition of $|m|(G)$, there exist a finite $\mathrm{Ba}\left(Z_{B}\right)$-partition $\left\{F_{i}\right\}$ of $G$ and $s_{i} \in E$, with $\left\|s_{i}\right\| \leqslant 1$, such that $\Sigma m\left(F_{i}\right) s_{i}>|m|(G)-\epsilon$. By the regularity of each $m s_{i}$, there are $B$-zero sets $Z_{i} \subset F_{i}$ such that $\Sigma m\left(Z_{i}\right) s_{i}>|m|(G)$ $-\epsilon$. The $B$-zero set $Z=\bigcup Z_{i}$ is contained in $G$. Moreover, $|m|(Z) \geqslant \Sigma m\left(Z_{i}\right) s_{i}$ $>|m|(G)-\epsilon$ which proves the regularity of $|m|$.

To finish the proof it remains to show that $|m|$ is countably-additive. To this end, consider a sequence $\left\{F_{i}\right\}$ of disjoint members of $\mathrm{Ba}\left(Z_{B}\right)$ and let $G=$ $\bigcup_{i} F_{i}$. Since $|m|$ is monotone and finitely-additive, we have

$$
|m|(G) \geqslant|m|\left(\bigcup_{i=1}^{n} F_{i}\right)=\sum_{i=1}^{n}|m|\left(F_{i}\right)
$$

for all $n$ and hence $|m|(G) \geqslant \sum_{i=1}^{\infty}|m|\left(F_{i}\right)$. On the other hand, let $\epsilon>0$ be given. There exist a $\operatorname{Ba}\left(Z_{B}\right)$-partition $G_{1}, \ldots, G_{N}$ of $G$, and $s_{i} \in E$, with $\left\|s_{i}\right\| \leqslant 1$, such that $\Sigma_{1}^{N} m\left(G_{i}\right) s_{i}>|m|(G)-\epsilon$. For each $i$ we have $m\left(G_{i}\right) s_{i}=\Sigma_{n=1}^{\infty} m\left(G_{i} \cap F_{n}\right) s_{i}$. Moreover,

$$
\sum_{n=1}^{\infty} \sum_{i=1}^{N}\left|m\left(G_{i} \cap F_{n}\right) s_{i}\right| \leqslant \sum_{n=1}^{\infty}|m|\left(F_{n}\right) \leqslant|m|(G)
$$

Hence

$$
\begin{aligned}
|m|(G)-\epsilon & <\sum_{i=1}^{N} m\left(G_{i}\right) s_{i}=\sum_{i=1}^{N} \sum_{n=1}^{\infty} m\left(G_{i} \cap F_{n}\right) s_{i} \\
& =\sum_{n=1}^{\infty} \sum_{i=1}^{N} m\left(G_{i} \cap F_{n}\right) s_{i}<\sum_{n=1}^{\infty}|m|\left(F_{n}\right) \leqslant|m|(G) .
\end{aligned}
$$

Since $\epsilon>0$ was arbitrary, we conclude that $|m|(G)=\Sigma_{n=1}^{\infty}|m|\left(F_{n}\right)$. This completes the proof.

THEOREM 4.2. If $m \in M_{\tau}\left(\mathrm{Bo}(X), E^{\prime}\right)$, then $|m| \in M_{\tau}(X)$.

Proof. Using an argument similar to that of Theorem 4.1, we show that $|m|$ is a regular Borel measure on $\operatorname{Bo}(X)$. It remains to show that $|m|$ is $\tau$-additive. By the note at the beginning of $\S 4$, it suffices to show that $|m|\left(Z_{\alpha}\right) \rightarrow 0$ for each net $\left\{Z_{\alpha}\right\}$ of $B$-zero sets which decreases to the empty set. So, let $\left\{Z_{\alpha}\right\}$ be such a net. For each $\alpha$ there exists a zero set $\hat{Z}_{\alpha}$ in $Y$ such that $Z_{\alpha}=\hat{Z}_{\alpha} \cap X$. Define $\bar{m}: \operatorname{Bo}(Y) \rightarrow E^{\prime}$ by $\bar{m}(F)=m(F \cap X)$. For each $s \in E$, the function $\bar{m} s: \operatorname{Bo}(Y) \rightarrow R,(\bar{m} s)(F)=(m s)(F \cap X)$ is in $M_{\tau}(Y)$ since $m s$ is $\tau$-additive. It 
now follows easily that $\bar{m} \in M_{\tau}\left(\mathrm{Bo}(Y), E^{\prime}\right)$ and that $|\bar{m}|(F)=|m|(F \cap X)$ for each Borel set $F$ in $Y$. Let $D$ denote the collection of all subsets $Z$ of $Y$ which are intersections of a finite number of $\hat{Z}_{\alpha}$ 's. Then $D$ is directed downwards to $G=\bigcap \hat{Z}_{\alpha}$. Hence $|\bar{m}|(G)=\lim _{Z \in D}|\bar{m}|(Z)$. Since $G \cap X=\varnothing$ we have $|m|(G)$ $=0$. Therefore, given $\epsilon>0$ there exists a $Z=\hat{Z}_{\alpha_{1}} \cap \cdots \cap \hat{Z}_{\alpha_{n}}$ in $D$ such that $|\bar{m}|(Z)<\epsilon$. Now, if $\alpha \geqslant \alpha_{1}, \ldots, \alpha_{n}$, then

$$
|m|\left(Z_{\alpha}\right) \leqslant|m|\left(Z_{\alpha_{1}} \cap \cdots \cap Z_{\alpha_{n}}\right)=|\bar{m}|(Z)<\epsilon .
$$

This proves that $\lim |m|\left(Z_{\alpha}\right)=0$ and the proof is complete.

We have analogous theorems for the elements in the spaces $M_{\sigma}\left(\mathrm{Ba}(Y), E^{\prime}\right)$ and $M_{\tau}\left(\mathrm{Bo}(Y), E^{\prime}\right)$.

Next we will define integrals with respect to measures belonging to one of the spaces defined above. The integration process which we will employ is a generalization, to the vector case, of the process of Aleksandrov. It is one of the many integration processes defined by McShane [17].

Let $m \in M_{\sigma}\left(\mathrm{Ba}\left(Z_{B}\right), E^{\prime}\right), G \in \mathrm{Ba}\left(Z_{B}\right)$, and $f \in A$. Consider the collection $D$ of all $\alpha=\left\{F_{1}, \ldots, F_{n} ; x_{1}, \ldots, x_{n}\right\}$ where $\left\{F_{i}\right\}$ is a $\mathrm{Ba}\left(Z_{B}\right)$ partition of $G$ and $x_{i} \in F_{i}$. For $\alpha_{1}, \alpha_{2} \in D$ we write $\alpha_{1} \geqslant \alpha_{2}$ iff the partition of $G$ in $\alpha_{1}$ is a refinement of the partition of $G$ in $\alpha_{2}$. Then $(D, \geqslant)$ is a directed set. For each $\alpha=\left\{F_{1}, \ldots, F_{n} ; x_{1}, \ldots, x_{n}\right\}$ in $D$ we define $w_{\alpha}=\Sigma m\left(F_{i}\right) f\left(x_{i}\right)$. We will show that $\left\{w_{\alpha}\right\}$ is a Cauchy net in $R$ and hence convergent. Indeed, let $\epsilon>0$ be given. We may assume, without loss of generality, that $\|m\| \leqslant 1$. For each $x \in X$, let $V_{x}=\{y \in X:\|f(x)-f(y)\|<\epsilon\}$. Then $V_{x}$ is a $B$-cozero set and hence in $\mathrm{Ba}\left(Z_{B}\right):$ If $W=\{s \in E:\|s\|<\epsilon\}$, then $V_{x}=f^{-1}(f(x)+W)$. Since $f(X)$ is totally bounded, there are $x_{1}, \ldots, x_{N}$ in $X$ such that $f(X) \subset \bigcup_{i=1}^{N}\left(f\left(x_{i}\right)+W\right)$. Thus $X=\bigcup_{i=1}^{N} V_{x_{i}}$. Let $G_{i}^{\prime}=V_{x_{i}} \cap G$. Define $G_{1}=G_{1}^{\prime}$ and $G_{n+1}=G_{n+1}^{\prime}-$ $\bigcup_{i}^{n} G_{i}, n=1, \ldots, N-1$. Keeping those $G_{i}$ which are not empty we get a $\mathrm{Ba}\left(Z_{B}\right)$ partition $\left\{F_{1}, \ldots, F_{n}\right\}$ of $G$ with the property that $\|f(x)-f(y)\| \leqslant 2 \epsilon$ if $x, y$ are in the same $F_{i}$. Pick $x_{i} \in F_{i}$ and let $\alpha_{0}=\left\{F_{1}, \ldots, F_{n} ; x_{1}, \ldots, x_{n}\right\}$. If $\alpha_{1}, \alpha_{2}$ are in $D$ with $\alpha_{1}, \alpha_{2} \geqslant \alpha_{0}$, then

$$
\left|w_{\alpha_{1}}-w_{\alpha_{2}}\right| \leqslant\left|w_{\alpha_{1}}-w_{\alpha_{0}}\right|+\left|w_{\alpha_{0}}-w_{\alpha_{2}}\right| \leqslant 2 \epsilon|m|(G)+2 \epsilon|m|(G) \leqslant 4 \epsilon .
$$

This proves that the net $\left\{w_{\alpha}\right\}$ is a Cauchy net and hence convergent. We define $\int_{G} f d m=\lim w_{\alpha}$. It can be shown easily that, for disjoint $F_{1}$ and $F_{2}$ in $\operatorname{Ba}\left(Z_{B}\right)$ and $G=F_{1} \cup F_{2}$, we have $\int_{G} f d m=\int_{F_{1}} f d m+\int_{F_{2}} f d m$. Moreover we have the following easily established

LEMma 4.3. (a) The map $f \rightarrow \int_{G} f d m$ is linear on $A$.

$$
\left|\int_{G} f d m\right| \leqslant \int_{G}\|f(x)\| d|m|(x) \leqslant\|f\||m|(G) \text { for all } f \in A .
$$


We similarly define integrals of functions in $A$ with respect to members of $M_{\tau}\left(\mathrm{Bo}(X), E^{\prime}\right)$.

LEMMA 4.4. Let $m$ be a bounded, real-valued, countably additive measure on $\mathrm{Ba}\left(Z_{B}\right)$. Then $m \in M_{\sigma}(B)$.

Proof. We first show that for any $B$-zero set $Z$ and any $\epsilon>0$ there exists a $B$-cozero set $V$, containing $Z$, such that $|m|(Z)>|m|(V)-\epsilon$. Indeed, if $Z$ is a $B$-zero set, there exists $f \geqslant 0$ in $B$ such that $Z=f^{-1}\{0\}$. For each positive integer $n$, we set $V_{n}=\{x: f(x)<1 / n\}$. Then $V_{n}$ is a $B$-cozero set and the sequence $\left\{V_{n}\right\}$ decreases to $Z$. Since $|m|$ is countably additive, we have $|m|(Z)=$ $\lim |m|\left(V_{n}\right)$ which implies our claim. Thus every $B$-zero set belongs to the family $\Sigma$ of all subsets $G$ of $X$ with the following property: Given $\epsilon>0$ there exist a $B$-zero set $Z$ and a $B$-cozero set $V$, with $Z \subset G \subset V$, such that $|m|(V-Z)<\epsilon$. The family $\Sigma$ is a $\sigma$-algebra which contains all $B$-zero sets and hence $\operatorname{Ba}\left(Z_{B}\right) \subset \Sigma$. This implies that $m$ is regular with respect to the family of all $B$-zero sets. The lemma is proved.

LEMMA 4.5. Let $m \in M_{\tau}\left(\mathrm{Bo}(X), E^{\prime}\right)$ and let $\mu$ denote the restriction of $m$ to $\mathrm{Ba}\left(Z_{B}\right)$. Then $\mu \in M_{\sigma}\left(\mathrm{Ba}\left(Z_{B}\right), E^{\prime}\right)$ and $\int_{G} f d m=\int_{G} f d \mu$ for each $f \in A$.

PROoF. In view of the preceding lemma, the restriction $\left.m s\right|_{\mathrm{Ba}\left(Z_{B}\right)}$ belongs to $M_{\sigma}(B)$ for all $s \in E$. Now, it follows that $\mu \in M_{\sigma}\left(\mathrm{Ba}\left(Z_{B}\right), E^{\prime}\right)$. If we look at the proof of the existence of $\int_{G} f d \mu$ and $\int_{G} f d m$ we can see that $\int_{G} f d m$ and $\int_{G} f d \mu$ coincide.

Integrals of functions in $C$, with respect to members of $M_{\sigma}\left(\mathrm{Ba}(Y), E^{\prime}\right)$ and $M_{\tau}\left(\mathrm{Bo}(Y), E^{\prime}\right)$, are defined similarly.

LEMmA 4.6. If $m_{1}, m_{2} \in M_{\tau}\left(\operatorname{Bo}(Y), E^{\prime}\right)$ are such that $\int_{Y} \hat{f} d m_{1}=\int_{Y} \hat{f} d m_{2}$ for all $f \in A$, then $m_{1}=m_{2}$.

Proof. Let $s \in E$. For each $f \in B$, we have

$$
\int_{Y} \hat{f} d\left(m_{1} s\right)=\int_{Y} \hat{f s} d m_{1}=\int_{Y} \hat{f} s d m_{2}=\int_{Y} \hat{f} d\left(m_{2} s\right) .
$$

By the uniqueness part of the Riesz representation theorem, we have $m_{1} s=m_{2} s$. This, being true for all $s \in E$, implies that $m_{1}=m_{2}$.

For a proof of the following theorem see Wells [25].

THEOREM 4.7. Let $\phi$ be a linear functional on $C$. Then $\phi$ is continuous with respect to the uniform norm topology iff there exists $m \in M_{\tau}\left(\mathrm{Bo}(Y), E^{\prime}\right)$ such that $\phi(\hat{f})=\int_{Y} \hat{f} d m$ for all $\hat{f} \in C$. Moreover, $\|\phi\|=\|m\|$.

If $m \in M_{\tau}\left(\mathrm{Bo}(Y), E^{\prime}\right)$ and $m_{1}=\left.m\right|_{\mathrm{Ba}(Y)}$ then $m_{1} \in M_{\sigma}\left(\mathrm{Ba}(Y), E^{\prime}\right)$ and $\int_{Y} \hat{f} d m_{1}=\int_{Y} \hat{f} d m$ for all $f \in A$. Furthermore, $\left\|m_{1}\right\|=\|m\|$. To prove the last equality, consider the linear map $\phi: C \rightarrow R, \phi(\hat{f})=\int_{Y} \hat{f} d m=\int_{Y} \hat{f} d m_{1}$. By 4.7 
we have $\|\phi\|=\|m\|$. Also $\|\phi\| \leqslant\left\|m_{1}\right\|$ since $|\phi(\hat{f})|=\left|\int_{Y} \hat{f} d m_{1}\right| \leqslant\|\hat{f}\|\left\|m_{1}\right\|$. Since $\|m\| \geqslant\left\|m_{1}\right\|$, it follows that $\|\phi\|=\left\|m_{1}\right\|=\|m\|$. Moreover, the inequality $\left|m_{1}\right|(G) \leqslant|m|(G)$, together with $\left|m_{1}\right|(Y)=|m|(Y)$, implies that $\left|m_{1}\right|=\left.|m|\right|_{\mathrm{Ba}(Y)}$.

Let now $\phi \in A^{\prime}$. Define $\hat{\phi}: C \rightarrow R, \hat{\phi}(\hat{f})=\phi(f)$. Clearly $\hat{\phi} \in C^{\prime}$. Let $m=\hat{m}_{\phi}$ be the element of $M_{\tau}\left(\mathrm{Bo}(Y), E^{\prime}\right)$ that corresponds to $\hat{\phi}$ by Theorem 4.7.

LEMMA 4.8. For a $Q \in \Omega$, the following are equivalent:

(1) $\phi \in\left(A, \beta_{Q}\right)^{\prime}$.

(2) $|m|(Q)=0$.

Proof. (1) $\rightarrow(2)$. By regularity it suffices to show that $m(G) s=0$ for each closed set $G$ in $Y$ contained in $Q$ and each $s \in E,\|s\| \leqslant 1$. So, let $G$ be such a set and $s \in E$ with $\|s\| \leqslant 1$. There exists an open set $O$ in $Y$ containing $G$ and such that $|m s|(O-G)<\epsilon\left(\epsilon>0\right.$ arbitrary). Since $\phi$ is $\beta_{Q}$-continuous, there exist $g \in B_{Q}$ and $K>0$ such that $|\phi(f)| \leqslant K$ for all $f \in A$ with $\|g f\| \leqslant 1$. Choose $n>0$ so that $K / n<\epsilon$. Set

$$
O_{1}=\{x \in Y:|\hat{g}(x)|<1 / n\} \text { and } O_{2}=O_{1} \cap O
$$

Clearly $G \subset O_{2}$ and $|\dot{m s}|\left(O_{2}-G\right)<\epsilon$. Choose $h \in B, 0 \leqslant h \leqslant 1, \hat{h}=1$ on $G$ and $\hat{h}=0$ on the complement of $O_{2}$. Let $f=n h s$. Since $\|g f\| \leqslant 1$, we have $|\phi(h s)| \leqslant K / n<\epsilon$. But

$$
|\phi(h s)|=\left|m(F) s+\int_{O_{2}-G} \hat{h} s d m\right| \geqslant|m(F) s|-\epsilon .
$$

Thus $|m(G) s| \leqslant 2 \epsilon$ which proves that $m(G) s=0$ and (2) follows.

$(2) \rightarrow(1)$. Suppose that $|m|(Q)=0$ and let $r>0$. Choose an open set $V$ in $Y$ with $|m|(V)<1 /(2 r), Q \subset V$. There exists $g \in B_{Q}$ such that $\hat{g}=1$ on the complement of $V$. Set $W=\{f \in A:\|g f\| \leqslant 1 / 2\|m\|\}$. Then $W \cap U_{r} \subset H$ where $H=\{f \in A:|\phi(f)| \leqslant 1\}$ and $U_{r}=\{f \in A:\|f\| \leqslant r\}$. This shows that $H$ is a $\beta_{Q}$-neighborhood of zero and hence $\phi$ is $\beta_{Q}$-continuous.

THEOREM 4.9. Let $\phi \in A^{\prime}$ and let $m \in M_{\tau}\left(\mathrm{Bo}(Y), E^{\prime}\right)$ be such that $\phi(f)=$ $\int_{Y} \hat{f} d m$ for all $f \in A$. Then:

(1) $\phi \in(A, \beta)^{\prime}$ iff $|m|(Q)=0$ for all $Q \in \Omega$.

(2) $\phi \in\left(A, \beta_{1}\right)^{\prime}$ iff $|m|(Z)=0$ for all $Z \in \Omega_{1}$.

Proof. It follows from the preceding lemma and from the fact that $\phi$ is $\beta$-continuous iff $\phi$ is $\beta_{Q}$-continuous for all $Q \in \Omega$, and $\phi$ is $\beta_{1}$-continuous iff $\phi$ is $\beta_{Q}$-continuous for all $Q \in \Omega_{1}$.

Let now $\hat{m} \in M_{\tau}\left(\mathrm{Bo}(Y), E^{\prime}\right)$ be such that $|\hat{m}|(Q)=0$ for all $Q \in \Omega$. By the regularity of $|\hat{m}|$, we have $|\hat{m}|(G)=0$ for each Borel set $G$ in $Y$ disjoint from $X$. Define $m: \operatorname{Bo}(X) \rightarrow E^{\prime}$ by $m(G \cap X)=\hat{m}(G)$ for each $G$ in $\operatorname{Bo}(Y)$. This 
gives us a well-defined function on $\operatorname{Bo}(X)$. The proof of the following is straightforward and we omit it.

LEMMA 4.10. (1) $m \in M_{\tau}\left(\mathrm{Bo}(X), E^{\prime}\right)$.

(2) $|m|(G \cap X)=|\hat{m}|(G)$ for each $G$ in $\mathrm{Bo}(Y)$.

(3) $\int_{X} f d m=\int_{Y} \hat{f} d \hat{m}$ for all $f \in A$.

Similarly, if $\hat{m}_{1} \in M_{\sigma}\left(\mathrm{Ba}(Y), E^{\prime}\right)$ is such that $\left|\hat{m}_{1}\right|(Z)=0$ for each $Z \in \Omega_{1}$, then the function $m_{1}: \mathrm{Ba}\left(Z_{B}\right) \rightarrow E^{\prime}, m_{1}(G \cap X)=\hat{m}_{1}(G)$ for all $G \in \mathrm{Ba}(Y)$, is well defined and the following is true.

LEMMA 4.11. (1) $m_{1} \in M_{\sigma}\left(\mathrm{Ba}\left(Z_{B}\right), E^{\prime}\right)$.

(2) $\left|m_{1}\right|(G \cap X)=\left|\hat{m}_{1}\right|(G)$ for each $G$ in $\mathrm{Ba}(Y)$.

(3) $\int_{X} f d m_{1}=\int_{Y} \hat{f} d \hat{m}_{1}$ for each $f \in A$.

An element $\phi$ of the uniform dual $B^{\prime}$ of $B$ is called $\tau$-additive iff $\phi\left(f_{\alpha}\right) \rightarrow 0$ for each net $\left\{f_{\alpha}\right\}$ in $B$ which decreases pointwise to zero. Let $L_{\tau}(B)$ denote the collection of all $\tau$-additive members of $B$.

LEMma 4.12. The map $m \rightarrow \phi$ defined by the formula $\phi(f)=\int f d m$ for all $f \in B$ establishes an isomorphism between the spaces $M_{\tau}(X)$ and $L_{\tau}(B)$.

Proof. By LeCam $\left[16\right.$, p. 214], every $\tau$-additive member of $B^{\prime}$ has a unique extension to a $\tau$-additive functional on the space $C^{b}(X)$ of all bounded continuous real-valued functions on $X$. By Varandarajan [24] and by Kirk [13, Theorem 1.12], the space of $\tau$-additive functionals on $C^{b}(X)$ is isomorphic to the space $M_{\tau}(X)$ via the isomorphism $m \rightarrow \phi, \phi(f)=\int f d m$ for all $f \in C^{b}(X)$. Hence the result follows.

LEMmA 4.13. Let $m \in M_{\tau}(X)$. Define $\bar{m}$ on $\operatorname{Bo}(Y)$ by $\bar{m}(G)=m(G \cap X)$. Then $\bar{m} \in \mathrm{Bo}(Y)$.

Proof. By (4.12), the linear functional $\phi$, defined on $B$ by $\phi(f)=\int f d m$, is $\tau$-additive. Define $\hat{\phi}$ on the space $C(Y)=\{\hat{f}: f \in B\}$ by $\hat{\phi}(\hat{f})=\phi(f)$. Then $\hat{\phi}$ is in the uniform dual of $C(Y)$. By the Riesz representation theorem there exists $\mu \in M_{\tau}(Y)$ such that $\hat{\phi}(\hat{f})=\int \hat{f} d \mu$ for all $f \in B$. By an argument similar to that employed by Knowles [14, Theorem 2.4], we show that $|\mu|(G)=0$ for each Borel subset $G$ of $Y$ which is disjoint from $X$. Define $m_{1}$ on $\operatorname{Bo}(X)$ by $m_{1}(G \cap X)=\mu(G)$ for all $G \in \operatorname{Bo}(Y)$. It is easy to see that $m_{1}$ is a well-defined element of $M_{\tau}(X)$ and $\int_{X} f d m_{1}=\int_{Y} \hat{f} d \mu=\phi(f)=\int_{X} f d m$ for all $f \in B$. By 4.12 we have $m=m_{1}$. Since for $G \in \operatorname{Bo}(Y), \mu(G)=m_{1}(G \cap X)=m(G \cap X)$, it follows that $\hat{m}=\mu \in M_{\tau}(Y)$. This completes the proof.

LEMMA 4.14. If $m$ and $m_{1}$ are both in $M_{\sigma}(B)$ and if $\int f d m=\int f d m_{1}$ for all $f \in B$, then $m=m_{1}$. 
Proof. Let $Z$ be a $B$-zero set. There exists a sequence $\left\{f_{n}\right\}$ in $B$ which decreases pointwise to the characteristic function $\chi_{Z}=g$ of $Z$. Thus

$$
m(Z)=\int g d m=\lim \int f_{n} d m=\lim \int f_{n} d m_{1}=m_{1}(Z) .
$$

The result now follows from the regularity of $m$ and $m_{1}$.

Lemma 4.15. Let $m \in M_{\sigma}(B)$. Define $\bar{m}$ on $\operatorname{Ba}(Y)$ by $\bar{m}(G)=m(G \cap X)$ for all $G \in \mathrm{Ba}(Y)$. Then $\bar{m} \in M_{\sigma}(Y)$.

Proof. Let $\mu \in M_{\tau}(Y)$ be such that $\int_{Y} \hat{f} d \mu=\int_{X} f d m$ for all $f \in B$. Let $\mu_{1}=\left.\mu\right|_{\mathrm{Ba}(Y)}$. Then $\mu_{1} \in M_{\sigma}(Y)$ and $\int_{X} f d m=\int_{Y} \hat{f} d \mu_{1}$ for all $f \in B$. Since the functional $f \rightarrow \int f d m$ is $\sigma$-additive on $B$ (i.e. $\int f_{n} d m \rightarrow 0$ for each sequence $\left\{f_{n}\right\}$ in $B$ which decreases pointwise to zero) it follows, as in the proof of Theorem 2.1 of Knowles [14], that $\left|\mu_{1}\right|(G)=0$ for each Baire set $G$ in $Y$ which is disjoint from $X$. Define $m_{1}: \mathrm{Ba}\left(Z_{B}\right) \rightarrow R, m_{1}(G \cap X)=\mu_{1}(F)$ for each Baire set $G$ in $Y$. Then $m_{1}$ is a well-defined member of $M_{\sigma}(B)$ and $\int f d m_{1}=\int \hat{f} d \mu_{1}=$ $\int f d m$ for all $f \in B$. By Lemma 4.14 we have $m_{1}=\mu_{1}$. Thus $m=\mu_{1} \in M_{\sigma}(Y)$.

Now using 4.13 and 4.15 we easily get the following result.

Lemma 4.16. Let $m \in M_{\tau}\left(\mathrm{Bo}(X), E^{\prime}\right)$ and $m_{1} \in M_{\sigma}\left(\mathrm{Ba}\left(Z_{B}\right), E^{\prime}\right)$. Define $\hat{m}$ and $\hat{m}_{1}$ on $\operatorname{Bo}(Y)$, respectively, by $\hat{m}(Q)=m(Q \cap X), \hat{m}_{1}(G)=m_{1}(G \cap X)$. Then:

(1) $\hat{m} \in M_{\tau}\left(\mathrm{Bo}(Y), E^{\prime}\right)$ and $\hat{m}_{1} \in M_{\sigma}\left(\mathrm{Ba}(Y), E^{\prime}\right)$.

(2) $|\hat{m}|(Q)=|m|(Q \cap X)$ for all $Q \in \operatorname{Bo}(Y)$, and $\left|\hat{m}_{1}\right|(Q)=\left|m_{1}\right|(G \cap X)$ for all $G \in \mathrm{Ba}(Y)$.

(3) $\int_{X} f d m=\int_{Y} \hat{f} d \hat{m}$ and $\int_{X} f d m_{1}=\int_{Y} \hat{f} d \hat{m}_{1}$ for all $f \in A$.

LEMMA 4.17. Every $m \in M_{\sigma}\left(\mathrm{Ba}(Y), E^{\prime}\right)$ has a unique extension to $a \mu$ in $M_{\tau}\left(\mathrm{Ba}(Y), E^{\prime}\right)$.

Proof. Define $\phi$ on $C$ by $\phi(\hat{f})=\int_{\gamma} \hat{f} d m$. Then $\phi \in C^{\prime}$. By 4.7 there exists a unique $\mu$ in $M_{\tau}\left(\operatorname{Bo}(Y), E^{\prime}\right)$ such that $\phi(\hat{f})=\int \hat{f} d \mu$ for all $f \in A$. Let

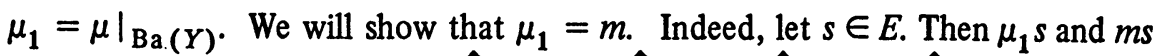
are both in $M_{\sigma}(Y)$. Moreover $\int \hat{f} d(m s)=\int \hat{f} s d m=\int \hat{f} s d \mu_{1}=\int \hat{f} d\left(\mu_{1} s\right)$ for all $f \in B$. It follows that $m s=\mu_{1} s$ for all $s \in E$ and hence $m=\mu_{1}$. Since $\mu \in$ $M_{\sigma}\left(\mathrm{Bo}(Y), E^{\prime}\right)$ the result follows.

Combining Lemmas 4.6, 4.17 and 4.16 we get

LEMMA 4.18. If $m_{1}, m_{2} \in M_{\tau}\left(\mathrm{Bo}(X), E^{\prime}\right)\left[m_{1}, m_{2} \in M_{\sigma}\left(\mathrm{Ba}\left(Z_{B}\right), E^{\prime}\right)\right]$ are such that $\int_{X} f d m_{1}=\int_{X} f d m_{2}$ for all $f \in A$, then $m_{1}=m_{2}$.

We are now in a position to identify the dual spaces of $(A, \beta),\left(A, \beta_{1}\right)$ and $\left(A, \beta_{F}\right)$. 
THEOREM 4.19. Let $\phi \in A^{\prime}$. Then:

(1) $\phi$ is $\beta$-continuous iff there exists $m \in M_{\tau}\left(\mathrm{Bo}(X), E^{\prime}\right)$ such that $\int f d m=$ $\phi(f)$ for all $f \in A$.

(2) $\phi$ is $\beta_{1}$-continuous iff there exists $m \in M_{\sigma}\left(\mathrm{Ba}\left(Z_{B}\right), E^{\prime}\right)$ such that $\phi(f)=\int f d m$ for all $f \in A$.

Furthermore, the $m$ that corresponds to a $\beta$-continuous ( $\beta_{1}$-continuous) member $\phi$ of $A^{\prime}$ is unique and $\|\phi\|=\|m\|$.

Proof. (1) Suppose that $\phi$ is $\beta$-continuous. Let $\hat{m}$ be the element of $M_{\tau}\left(\mathrm{Bo}(Y), E^{\prime}\right)$ with the property that $\phi(f)=\int \hat{f} d \hat{m}$ for all $f \in A$. Define $m$ on $\operatorname{Bo}(X)$ by $m(G \cap X)=\hat{m}(G)$ for all $G \in \operatorname{Bo}(Y)$. This gives us an element $m$ of $M_{\tau}\left(\mathrm{Bo}(X), E^{\prime}\right)$ by 4.9 and 4.10. Moreover, by 4.10, $\int_{X} f d m=\int_{Y} \hat{f} d \hat{m}=\phi(f)$ for all $f \in A$. Also $\|\phi\|=\|\hat{m}\|=\|m\|$. Conversely, let $m \in M_{\tau}\left(\operatorname{Bo}(X), E^{\prime}\right)$ be such that $\phi(f)=\int f d m$ for all $f \in A$. Define $\hat{m}$ on $\operatorname{Bo}(Y)$ by $\hat{m}(G)=m(G \cap X)$. By 4.16, we have $m \in M_{\tau}\left(\operatorname{Bo}(Y), E^{\prime}\right)$ and $\int_{X} f d m=\int_{Y} \hat{f} d \hat{m}$ for all $f \in A$. Since $|\hat{m}|(Q)=|m|(Q \cap X)=0$ for all $Q \in \Omega$, we have $\phi \in(A, \beta)^{\prime}$ by 4.9. Finally the uniqueness of $m$ follows from 4.18.

(2) The proof is similar to that of (1).

THEOREM 4.20. For $a \phi \in A^{\prime}$ the following are equivalent:

(1) $\phi \in\left(A, \beta_{F}\right)^{\prime}$.

(2) There exists $m \in M_{\tau}\left(\mathrm{Bo}(X), E^{\prime}\right)$ such that

(a) $\phi(f)=\int f d m$ for all $f \in A$,

(b) given $\epsilon>0$ there exists $G \in F$ with $|m|(X-G)<\epsilon$.

Proof. (2) $\rightarrow$ (1). Let $\epsilon>0$ be given. Choose $G$ in $F$ with $|m|(X-G)$ $<\epsilon / 2$. Let $\delta>0$ be such that $2 \delta\|m\|<\epsilon$. If $f \in A,\|f\| \leqslant 1,\|f\|_{G} \leqslant \delta$, then

$$
|\phi(f)| \leqslant\left|\int_{G} f d m\right|+\left|\int_{X-G} f d m\right| \leqslant \delta|m|(G)+|m|(X-G) \leqslant \epsilon .
$$

Hence $\phi \in\left(A, \beta_{F}\right)^{\prime}$ by 3.7 .

$(1) \rightarrow(2)$. Since $\beta_{F} \leqslant \beta$, we have $\phi \in(A, \beta)^{\prime}$. Hence there exists $m \in$ $M_{\tau}\left(\mathrm{Bo}(X), E^{\prime}\right)$ such that $\phi(f)=\int f d m$ for all $f$ in $A$. Define $\hat{m}$ on $\operatorname{Bo}(Y)$ by $\hat{m}(G)=m(G \cap X)$. By $4.16, \hat{m} \in M_{\tau}\left(\mathrm{Bo}(Y), E^{\prime}\right)$. Let $\epsilon>0$ be given. By 3.7 there exist $G$ in $F$ and $\delta>0$ such that $|\phi(f)| \leqslant \epsilon_{1}=\epsilon / 3$ for all $f$ in $W=$ $\left\{h \in A:\|h\| \leqslant 1,\|h\|_{G} \leqslant \delta\right\}$. By the definition of $|\hat{m}|$ there exist a partition $F_{1}, \ldots, F_{n}$ of $Y-G$, into Borel sets, and $s_{i} \in E$, with $\left\|s_{i}\right\| \leqslant 1$, such that $\Sigma \hat{m}\left(F_{i}\right) s_{i}>|\hat{m}|(Y-G)-\epsilon_{1}=|m|(X-G)-\epsilon_{1}$. There are closed sets $G_{i}$ in $Y, G_{i} \subset F_{i}$, such that $\Sigma \hat{m}\left(G_{i}\right) s_{i}>|m|(X-G)-\epsilon_{1}$. Choose pairwise disjoint open sets $V_{i}, 1 \leqslant i \leqslant n, G_{i} \subset V_{i} \subset Y-G$, such that $\Sigma|\hat{m}|\left(V_{i}-G_{i}\right)<\epsilon_{1}$. For each $i, 1 \leqslant i \leqslant n$, choose $h_{i}$ in $B, 0 \leqslant h_{i} \leqslant 1, \hat{h}_{i}=1$ on $G_{i}$ and $\hat{h}_{i}=0$ on $Y-V_{i}$. Let $f=\Sigma h_{i} s_{i}$. Then $f \in W$ and hence $|\phi(f)| \leqslant \epsilon_{1}$. Since 


$$
\phi(f) \leqslant \sum \int_{G_{i}} s_{i} d \hat{m}+\sum \int_{V_{i}-G_{i}} \hat{f} d \hat{m} \geqslant \sum \hat{m}\left(G_{i}\right) s_{i}-\epsilon_{1} \geqslant|m|(X-G)-2 \epsilon_{1},
$$

it follows that $|m|(X-G) \leqslant 3 \epsilon_{1}=\epsilon$. The theorem is proved.

Definition. A subset $M_{0}$ of $M_{\tau}\left(\mathrm{Bo}(X), E^{\prime}\right)$ is called $F$-tight if $M_{0}$ is norm bounded and given $\epsilon>0$ there exists $G$ in $F$ with $|m|(X-G) \leqslant \epsilon$ for all $m$ in $M_{0}$.

Lemma 4.21. Let $\phi \in(A, \beta)^{\prime}$ and let $m$ be the corresponding element of $M_{\tau}\left(\operatorname{Bo}(X), E^{\prime}\right)$. Let $G \in F$ and $\epsilon>0$. The following are equivalent:

(1) $|m|(X-G) \leqslant \epsilon$.

(2) For all $f \in A$ with $\|f\| \leqslant 1$ and $\|f\|_{G}=0$ we have $|\phi(f)| \leqslant \epsilon$.

We omit the proof of this lemma since we can use an argument similar to that used in the implication (1) $\rightarrow$ (2) of Theorem 4.20.

For $H \subset L_{F}(A)$, let $M_{H}=\left\{m_{\phi}: \phi \in H\right\} \subset M_{\tau}\left(\mathrm{Bo}(X), E^{\prime}\right)$ where $m_{\phi}$ is the measure that corresponds to $\phi$.

THEOREM 4.22. For $H \subset L_{F}(A)$ the following are equivalent:

(1) $H$ is $\beta_{F}$-equicontinuous.

(2) (a) $H$ is norm bounded.

(b) Given $\epsilon>0$ there exists $G \in F$ such that $|\phi(f)| \leqslant \epsilon$ for all $\phi \in H$ and all $f \in A$ with $\|f\| \leqslant 1$ and $f=0$ on $G$.

(3) $M_{H}$ is F-tight.

Proof. By $4.21,(2)$ and (3) are equivalent.

(1) $\rightarrow$ (2). (a) The set $U_{1}=\{f \in A:\|f\| \leqslant 1\}$ is norm bounded and hence $\beta$-bounded. Since $H^{0}\left(=\right.$ polar of $H$ with respect to the pair $\left.\left\langle L_{F}(A), A\right\rangle\right)$ is a $\beta_{F}$-neighborhood of zero there exists $K>0$ such that $U_{1} \subset K H^{0}$. It follows that $\|\phi\| \leqslant K$ for all $\phi$ in $H$.

(b) Let $\epsilon>0$ be given. Since $\epsilon H^{0}$ is a $\beta_{F}$-neighborhood of zero there exist $G$ in $F$ and $\delta>0$ such that $W=\left\{f \in A:\|f\| \leqslant 1,\|f\|_{G} \leqslant \delta\right\} \subset \epsilon H^{0}$. Thus (b) follows.

(3) $\rightarrow$ (1). Let $d=\sup \left\{\left\|m_{\phi}\right\|: \phi \in H\right\}=\sup \left\{\left\|m_{\phi}\right\|: \phi \in H\right\}$. Given $r>0$ there exists $G \in F$ such that $\left|m_{\phi}\right|(X-F) \leqslant 1 /(2 r)$ for all $\phi \in H$. If $V=$ $\left\{f \in A:\|f\|_{G} \leqslant 1 /(2 d)\right\}$, then $V \cap U_{r} \subset H^{0}$, where $U_{r}=\{f \in A:\|f\| \leqslant r\}$. This shows that $H^{0}$ is a $\beta$-neighborhood of zero and this completes the proof.

5. In this section we will assume that $E$ is a Banach lattice. We write $f \geqslant g$ iff $f(x) \geqslant g(x)$ for all $x \in X$. Since the lattice operations are continuous, it is easy to verify that $A$, under the relation $\geqslant$, is a Banach lattice where for $f, g$ in $A$ we have

$$
(f \wedge g)(x)=f(x) \wedge g(x)
$$




$$
(f \vee g)(x)=f(x) \vee g(x), \text { and }|f|(x)=|f(x)|
$$

for all $x \in X$. For a $\phi \in A^{\prime}$ the $\phi^{+}, \phi^{-},|\phi|$ are the elements of $A^{\prime}$ which are defined on positive $f \in A$ by

$$
\begin{aligned}
& \phi^{+}(f)=\sup \{\phi(g): 0 \leqslant g \leqslant f\}, \\
& \phi^{-}(f)=-\inf \{\phi(g): 0 \leqslant g \leqslant f\}, \\
& |\phi|(f)=\sup \{|\phi(g)|:|g| \leqslant f\} .
\end{aligned}
$$

THEOREM 5.1. Each of the spaces $(A, \beta),\left(A, \beta_{1}\right)$ and $\left(A, \beta_{F}\right)$ is locally solid.

Proof. Let $W$ be a convex balanced $\beta$-neighborhood of zero. For each $Q \in \Omega$ there exists $g_{Q} \in B_{Q}$ such that $V_{Q}=\left\{f \in A:\left\|g_{Q} f\right\| \leqslant 1\right\} \subset W$. Each $V_{Q}$ is clearly solid. Hence the set $V=\bigcup\left\{V_{Q}: Q \in \Omega\right\}$ is solid. By Peressini $\left[18\right.$, p. 161], the convex balanced hull $V_{0}$ of $V$ is solid. Since $V_{0} \subset W$, the result follows for $(A, \beta)$. The proof for $\left(A, \beta_{1}\right)$ is similar. For the $\left(A, \beta_{F}\right)$ we observe that the class of sets of the form $\bigcap_{i=1}^{\infty}\left\{f \in A:\|f\|_{G_{i}} \leqslant a_{i}\right\}$, where $0<a_{i}$ $\rightarrow \infty$ and $G_{i} \in F$, consists of solid sets and is a $\beta_{F}$-base at zero.

Definitions. For a net $\left\{f_{\alpha}\right\}$ in $A$, we say that it decreases to zero, and write $f_{\alpha} \downarrow 0$, if for each $x \in X$ we have $\lim f_{\alpha}(x)=0$ and $0 \leqslant f_{\alpha}(x) \leqslant f_{\gamma}(x)$ whenever $\alpha \geqslant \gamma$. An element $\phi$ of $A^{\prime}$ is called $\tau$-additive if $\phi\left(f_{\alpha}\right) \rightarrow 0$ whenever $f_{\alpha} \downarrow 0$. We will say that $\phi$ is $\sigma$-additive if $\phi\left(f_{n}\right) \rightarrow 0$ for each sequence $\left\{f_{n}\right\}$ in $A$ which decreases to zero. The set of all $\sigma$-additive ( $\tau$-additive) members of $A^{\prime}$ will be denoted by $L_{\sigma}(A)\left(L_{\tau}(A)\right)$.

THEOREM 5.2. Each of the dual spaces $(A, \beta)^{\prime},\left(A, \beta_{1}\right)^{\prime}$ and $\left(A, \beta_{F}\right)^{\prime}$ forms a linear lattice ideal in the Riesz space $A^{\prime}$.

Proof. This follows easily from the fact that the spaces $(A, \beta),\left(A, \beta_{1}\right)$ and $\left(A, \beta_{F}\right)$ are locally solid.

THEOREM 5.3. The dual space of the space $(A, \beta)$ is the space $L_{\tau}(A)$.

Proof. Let $\phi \in A^{\prime}$ and let $m \in M_{\tau}\left(\operatorname{Bo}(Y), E^{\prime}\right)$ be such that $\phi(f)=\int \hat{f} d m$ for all $f \in A$. Suppose $\phi$ is $\beta$-continuous and let $f_{\alpha} \downarrow 0$. We want to show that $\phi\left(f_{\alpha}\right) \rightarrow 0$. Without loss of generality we may assume that $\left\|f_{\alpha}\right\| \leqslant 1$ for all $\alpha$. Let $\epsilon>0$. For each $\alpha$, set $Z_{\alpha}=\left\{x \in Y:\left\|\hat{f}_{\alpha}(x)\right\| \geqslant \epsilon\right\}$. Then $Z_{\alpha} \downarrow Q=\bigcap Z_{\alpha}$. Since $Q \in \Omega$ we have $|m|(Q)=0$ by 4.9. Since $|m|\left(Z_{\alpha}\right) \rightarrow|m|(Q)=0$, there exists $\alpha_{0}$ such that $|m|\left(Z_{\alpha}\right)<\epsilon$ for all $\alpha \geqslant \alpha_{0}$. Now, for $\alpha \geqslant \alpha_{0}$, we have

$$
\begin{aligned}
\left|\phi\left(f_{\alpha}\right)\right| & \leqslant\left|\int_{Z_{\alpha}} f d m\right|+\left|\int_{Y-Z_{\alpha}} \hat{f} d m\right| \\
& \leqslant|m|\left(Z_{\alpha}\right)+\epsilon\|m\| \leqslant \epsilon(1+\|m\|) .
\end{aligned}
$$

This shows that $\phi\left(f_{\alpha}\right) \rightarrow 0$ and $\phi$ is $\tau$-additive. 
Conversely, assume that $\phi$ is $\tau$-additive. Let $Q$ in $\Omega$ and $0 \leqslant s \in E,\|s\| \leqslant 1$. Choose an open set $O$ in $Y, Q \subset O$, such that $|m|(O-Q)<\epsilon(\epsilon>0$ arbitrary). The collection $D=\{h s: h \in B, 0 \leqslant h \leqslant 1, \hat{h}=1$ on $Q$ and $\hat{h}=0$ on $Y-O\}$ is downwards directed to zero. Hence there exists $h s$ in $D$ such that $|\phi(h s)|<\epsilon$. But

$$
|\phi(h s)| \geqslant\left|\int_{Q} s d m\right|-\left|\int_{O-Q} \hat{h} s d m\right| \geqslant|m(Q) s|-|m|(O-Q) \geqslant|m(Q) s|-\epsilon .
$$

Hence $|m(Q) s| \leqslant 2 \epsilon$. This proves that $m(Q) s=0$ for each $Q$ in $\Omega$ and each $s \in E,\|s\| \leqslant 1, s \geqslant 0$. Since $E$ is a lattice, we have $m(Q) s=0$ for all $s \in E$. Now the regularity of $m s$ and 4.9 complete the proof.

THEOREM 5.4. The dual of the space $\left(A, \beta_{1}\right)$ is the space $L_{\sigma}(A)$.

Proof. Let $\phi \in A^{\prime}$ and $m \in M_{\tau}\left(\operatorname{Bo}(Y), E^{\prime}\right)$ be such that $\phi(f)=\int_{Y} \hat{f} d m$ for all $f$ in $A$. Assume that $\phi$ is $\beta_{1}$-continuous and let $\left\{f_{n}\right\}$ be a sequence in $A$ that decreases to zero, We want to show that $\phi\left(f_{n}\right) \rightarrow 0$. We may assume without loss of generality that $\left\|f_{n}\right\| \leqslant 1$ for all $n$. Let $\epsilon>0$ and set $Z_{n}=\{x \in Y$ : $\left.\left\|\hat{f}_{n}(x)\right\| \geqslant \epsilon\right\}$. Then $Z_{n} \downarrow \bigcap Z_{n}=Z$ and $Z \in \Omega_{1}$. Since $\lim |m|\left(Z_{n}\right)=|m|(Z)$ $=0$, there exists $n_{0}$ such that $|m|\left(Z_{n}\right)<\epsilon$ if $n \geqslant n_{0}$. Now, if $n \geqslant n_{0}$, we have

$$
\left|\phi\left(f_{n}\right)\right| \leqslant\left|\int_{Z_{n}} \hat{f}_{n} d m\right|+\left|\int_{Y-Z_{n}} \hat{f}_{n} d m\right|<\epsilon+\epsilon\|m\| .
$$

This proves that $\phi\left(f_{n}\right) \rightarrow 0$ and hence $\phi$ is $\sigma$-additive. Conversely, assume $\phi \in$ $L_{\sigma}(A)$. Let $Z$ be in $\Omega_{1}$ and $s \in E$ with $s \geqslant 0$ and $\|s\| \leqslant 1$. Given $\epsilon>0$ there exists a cozero set $V$ containing $Z$ such that $|m|(V-Z)<\epsilon$. Let $g \in B, 0 \leqslant g$ $\leqslant 1$, be such that $Z=\hat{g}^{-1}\{0\}$. For each positive integer $n$, let $V_{n}=\{x \in Y$ : $\hat{g}(x)<1 / n\} \cap V$. Choose $h_{n} \in B, 0 \leqslant h_{n} \leqslant 1, \hat{h}_{n}=1$ on $Z$ and $\hat{h}=0$ on $Y-$ $V_{n}$. Let $h_{n}^{\prime}=h_{1} \wedge \ldots \wedge h_{n}$ and set $f_{n}=h_{n}^{\prime} s$. Then $f_{n} \downarrow 0$. Hence there exists $n$ such that $\left|\phi\left(f_{n}\right)\right|<\epsilon$. But

$$
\begin{aligned}
\left|\phi\left(f_{n}\right)\right| & \geqslant\left|\int_{Z} \hat{f}_{n} d m\right|-\left|\int_{V-Z} \hat{f_{n}} d m\right| \\
& \geqslant|m(Z) s|-|m|(V-Z) \geqslant|m(Z) s|-\epsilon .
\end{aligned}
$$

Thus $|m(Z) s| \leqslant 2 \epsilon$. This proves that $m(Z) s=0$. From this it follows that $m(Z) s=0$ for each $s \in E$ and all $Z$ in $\Omega_{1}$. Now the result follows from the regularity of $\left.m s\right|_{\mathrm{Ba}(Y)}$ and from 4.9.

THEOREM 5.5. Let $\tau$ be a locally convex Hausdorff topology on A for which the positive cone is normal. Then the following assertions are equivalent:

(1) $(A, \tau)^{\prime} \subset L_{\tau}(A)$.

(2) If $f_{\alpha} \downarrow 0$, then $f_{\alpha} \rightarrow 0$ in the $\tau$-topology. 
Proof. It is clear that (2) implies (1).

$(1) \rightarrow(2)$. By Schaefer [10, p. 219], $\tau$ is the topology of uniform convergence on the $\tau$-equicontinuous subsets of $(A, \tau)^{\prime+}=\left\{\phi \in(A, \tau)^{\prime}: \phi \geqslant 0\right\}$. Suppose now that $f_{\alpha} \downarrow 0$ and let $V$ be a $\tau$-neighborhood of zero. There exists a $\tau$-equicontinuous subset $H$ of $(A, \tau)^{\prime+}$ such that $H^{0} \subset V$. The set $H$ is relatively weakly compact. Every $f_{\alpha}$ defines a weakly continuous linear functional on $(A, \tau)^{\prime}$ by $\phi \rightarrow W_{\alpha}(f)=\phi\left(f_{\alpha}\right)$. If $\phi \in H$, then $W_{\alpha}(\phi) \downarrow 0$. Hence $W_{\alpha} \rightarrow 0$ uniformly on $H$ by Dini's theorem. It follows that there exists $\alpha_{0}$ such that $f_{\alpha} \in$ $H^{0} \subset V$ for all $\alpha \geqslant \alpha_{0}$. This proves that $f_{\alpha}^{\tau} \rightarrow 0$ and the proof is complete.

We have an analogous theorem for $L_{\sigma}(A)$ with a similar proof.

THEOREM 5.6. Let $\tau$ be a locally convex Hausdorff topology on A for which the positive cone is normal. The following are equivalent:

(1) The space $(A, \tau)^{\prime}$ is contained in $L_{\sigma}(A)$.

(2) $f_{n} \downarrow 0$ implies that $f_{n} \rightarrow 0$ in the $\tau$-topology.

CoRollary 5.7. (1) $f_{n} \downarrow 0$ implies that $f_{n} \rightarrow 0$ in the $\beta_{1}$-topology.

(2) $f_{\alpha} \downarrow 0$ implies that $f_{\alpha} \rightarrow 0$ in the $\beta$-topology.

THEOREM 5.8. Let $\tau$ be any one of the topologies $\beta, \beta_{1}, \beta_{F}$. If $W$ is a $\tau$-neighborhood of zero, then each of the sets $H_{1}=\left\{\phi^{+}: \phi \in W^{0}\right\}, H_{2}=\left\{\phi^{-}\right.$: $\left.\phi \in W^{0}\right\}$, and $H_{3}=\left\{|\phi|: \phi \in W^{0}\right\}$ is $\tau$-equicontinuous, where $W^{0}$ is the polar of $W$ in $(A, \tau)^{\prime}$.

Proof. Since $(A, \tau)$ is locally solid, $\tau$ is the topology of uniform convergence on the $\tau$-equicontinuous subsets of $(A, \tau)^{\prime+}$. Let $W_{1}$ be a solid $\tau$-neighborhood of zero contained in $W$ and let $H$ be a $\tau$-equicontinuous subset of $(A, \tau)^{\prime+}$ with $H^{0} \subset W_{1}$. Let $f \in H^{0}$ and $\phi \in W_{1}^{0} \subset H^{00}$. Since $W_{1}$ is solid we have

$$
\left|\phi^{+}(f)\right| \leqslant \phi^{+}(|f|)=\sup \{\phi(h): 0 \leqslant h \leqslant|f|\} \leqslant 1 .
$$

Thus $\phi^{+} \in H^{00}$. This shows that $H_{1} \subset H^{00}$. Similarly $H_{2}, H_{3} \subset H^{00}$ and the theorem is proved.

Throughout the remaining part of this paper $E$ will be assumed to be a Banach lattice with a unit element $e$ ( $e$ has the property that $-e \leqslant s \leqslant e$ iff $\|s\| \leqslant 1)$.

THEOREM 5.9. (1) Every weakly compact subset of $L_{\sigma}^{+}(A)$ is $\beta_{1}$-equicontinuous.

(2) Every weakly compact subset of $L_{\tau}^{+}(A)$ is $\beta$-equicontinuous.

(3) $\beta$ is the topology of uniform convergence on the weakly compact subsets of $L_{\tau}^{+}(A)$.

(4) $\beta_{1}$ is the topology of uniform convergence on the weakly compact subsets of $L_{\sigma}^{+}(A)$. 
Proof. (1) Let $H \subset L_{\sigma}^{+}(A)$ be weakly compact. The set $H^{0}$ is convex, balanced and absorbent. Let $r>0$. Set $\alpha=\sup \{\|\phi\|: \phi \in H\}$ and let $Z$ be in $\Omega_{1}$. There exists $f \in B, 0 \leqslant f \leqslant 1, Z=\hat{f}^{-1}\{0\}$. For each positive integer $n$, put $Z_{n}=\{x \in Y: \hat{f}(x) \geqslant 1 / n\}$. Choose $g_{n} \in B, 0 \leqslant g_{n} \leqslant 1, \hat{g}_{n}=1$ on $Z$ and $\hat{g}_{n}=0$ on $Z_{n}$. Let $h_{n}=g_{1} \wedge \cdots \wedge g_{n}$. Then $h_{n} e \downarrow 0$ and hence $\phi\left(h_{n} e\right) \downarrow 0$ for each $\phi$ in $H$. By Dini's theorem, $\phi\left(h_{n} e\right) \rightarrow 0$ uniformly on $H$. Hence there exists $n$ such that $\phi\left(h_{n} e\right)<1 /(2 r)$ for all $\phi \in H$. Let $g=1-h_{n}$ and set $V=\{h \in A:\|g h\| \leqslant 1 /(2 a)\}$. If $h \in V \cap U_{r}$, then $h_{n}|h| \leqslant r h_{n} e$. Hence for $h \in V \cap U_{r}$ and $\phi \in H$ we have $\left.\left.|\phi(h)| \leqslant \phi(|h| g)+\phi\left(h_{n}|h|\right) \leqslant\|\phi\|\|g h\|+r \phi\left(h_{n} e\right) \leqslant a(1 / 2 a)\right)+r(1 / 2 r)\right)=1$.

This shows that $V \cap U_{r} \subset H^{0}$. Since this happens for all $r>0$ and all $Z \in$ $\Omega_{1}$ we conclude that $H^{0}$ is a $\beta_{1}$-neighborhood of zero.

(2) Let $Q \in \Omega$. The set $D=\{g \in B: 0 \leqslant g \leqslant 1, \hat{g}=1$ on $Q\}$ is directed downwards to zero. From here on the proof is similar to that of (1).

(3) If $H \subset L_{\tau}^{+}(A)$ is weakly compact, then $H^{0}$ is a $\beta$-neighborhood of zero by (2). Conversely, let $W$ be a $\beta$-neighborhood of zero. Since $\beta$ is locally solid there exists a $\beta$-equicontinuous subset $H$ of $L_{\tau}^{+}(A)$ such that $H^{0} \subset W$. If $H_{1}$ is the weak closure of $H$ in $L_{\tau}(A)$, then $H_{1} \subset L_{\tau}^{+}(A)$ and $H_{1}$ is weakly compact. Moreover $H_{1}^{0} \subset H^{0} \subset W$. This proves (3).

(4) The proof is similar to that of (3).

Corollary 5.10. $\beta=\beta_{1}$ iff $L_{\tau}(A)=L_{\sigma}(A)$.

Using Dini's theorem and the Alaoglu-Bourbaki theorem (see Köthe [14, p. 248] ) we can easily show the following

THEOREM 5.11. (1) $A$ subset $H$ of $L_{\tau}^{+}(A)$ is $\sigma\left(L_{\tau}(A), A\right)$ relatively compact iff $\phi\left(f_{\alpha}\right) \rightarrow 0$ uniformly on $H$ for each net $\left\{f_{\alpha}\right\}$ in $A$ that decreases to zero.

(2) A subset $H$ of $L_{\sigma}^{+}(A)$ is $\sigma\left(L_{\sigma}(A), A\right)$ relatively compact iff $\phi\left(f_{n}\right)$ $\rightarrow 0$ uniformly on $H$ whenever $f_{n} \downarrow 0$.

Corollary 5.12. Let $H \subset L_{\tau}(A)\left(H \subset L_{\sigma}(A)\right)$. The following are equivalent:

(1) $\left\{\phi^{+}: \phi \in H\right\}$ and $\left\{\phi^{-}: \phi \in H\right.$ are both weakly relatively compact in $L_{\tau}(A)$ (in $\left.L_{\sigma}(A)\right)$.

(2) $\{|\phi|: \phi \in H\}$ is weakly relatively compact in $L_{\tau}(A)$ (in $L_{\sigma}(A)$ ).

THEOREM 5.13. The following are equivalent:

(1) $(A, \beta)$ is a Mackey space.

(2) If $H$ is a convex, balanced, weakly compact subset of $L_{\tau}(A)$, then $\{|\phi|: \phi \in H\}$ is weakly relatively compact in $L_{\tau}(A)$. 
(3) If $H$ is a convex, balanced, weakly compact subset of $L_{\tau}(A)$, then $\left\{\phi^{+}\right.$: $\phi \in H\}$ and $\left\{\phi^{-}: \phi \in H\right\}$ are both weakly relatively compact in $L_{\tau}(A)$.

Proof. By 5.12, (2) is equivalent to (3).

$(1) \rightarrow(3)$. Let $H$ be a weakly compact, convex, balanced subset of $L_{\tau}(A)$. By hypothesis $H^{0}$ is a $\beta$-neighborhood of zero. By 5.8 the sets $V_{1}=\left\{\phi^{+}\right.$: $\left.\phi \in H^{00}\right\}$ and $V_{2}=\left\{\phi^{-}: \phi \in H^{00}\right\}$ are weakly relatively compact in $L_{\tau}(A)$. Since $H \subset H^{00}$, (3) follows.

$(3) \rightarrow(1)$. Let $H$ be a convex balanced weakly compact subset of $L_{\tau}(A)$. By hypothesis and 5.9, the sets $H_{1}=\left\{\phi^{+}: \phi \in H\right\}$ and $H_{2}=\left\{\phi^{-}: \phi \in H\right\}$ are $\beta$-equicontinuous. Since $H^{0} \supset \frac{1}{2}\left(H_{1}^{0} \cap H_{2}^{0}\right)$, it follows that $H$ is $\beta$-equicontinuous. Hence $\beta$ is finer than the Mackey topology $m\left(A ; L_{\tau}(A)\right)$. Thus $\beta=$ $m\left(A, L_{\tau}(A)\right)$ since $(A, \beta)^{\prime}=L_{\tau}(A)$. This completes the proof.

We have an analogous theorem for the pair $\left\langle A, L_{\sigma}(A)\right\rangle$ and the topology $\beta_{1}$. The proof is similar.

\section{BIBLIOGRAPHY}

1. A. D. Aleksandrov, Additive set functions in abstract spaces, Mat. Sb. 8 (50) (1940), 307-348; ibid. 9 (51) (1941), 563-628. MR 2, 315; 3, 207.

2. W. M. Bogdanowicz, Representations of linear continuous functionals on the space $C(X, Y)$ of continuous functions from compact $X$ into locally convex $Y$, Proc. Japan Acad. 42 (1966), 1122-1127. MR 35 \#4365.

3. - An approach to the theory of integration generated by Daniell functionals and representation of linear continuous functionals, Math. Ann. 173 (1967), 34-52. MR 35 \#4364.

4. R. C. Buck, Bounded continuous functions on a locally compact space, Michigan Math. J. 5 (1958), 95-104. MR 21 \#4350.

5. J. B. Conway, The strict topology and compactness in the space of measures, Bull. Amer. Math. Soc. 72 (1966), 75-78. MR 32 \#4509.

6. J. B. Cooper, The strict topology and spaces with mixed topologies, Proc. Amer. Math. Soc. 30 (1971), 583-592. MR 44 \#2013.

7. N. Dinculeanu, Vector measures, Internat. Ser. of Monographs in Pure and Appl. Math., vol. 95, Pergamon Press, Oxford; VEB Deutscher Verlag, Berlin, 1967. MR 34 \#6011b.

8. I. Dobrakov, On integration in Banach spaces, Czechoslovak. Math. J. (20) 95 (1970), 511-536.

9. J. Dorroh, The localization of the strict topology via bounded sets, Proc. Amer. Math. Soc. 20 (1969), 413-414. MR 38 \#3721.

10. E. Hewitt, Linear functionals on the spaces of continuous functions, Fund. Math. 37 (1950), 161-189. MR 13, 147.

11. R. Giles, A generalization of the strict topology, Trans. Amer. Math. Soc. 161 (1971), 467-474. MR 43 \#7919.

12. A. Katsaras, Spaces of vector measures, Trans. Amer. Math. Soc. 206 (1975), 313-328.

13. R. B. Kirk, Locally compact, B-compact spaces, Nederl. Akad. Wetensch. Proc. Ser. A72 = Indag. Math. 31 (1969), 333-344. MR 41 \#9201.

14. J. D. Knowles, Measures on topological spaces, Proc. London Math. Soc. (3) 17 (1967), 139-156. MR 34 \#4441.

15. G. Köthe, Topologische linear Räume. I, Die Grundlehren der math. Wissenschaften, Band 107, Springer-Verlag, Berlin, 1960; English transl., Die Grundlehren der math. 
Wissenschaften, Band 159, Springer-Verlag, New York, 1969. MR 24 \#A411; 40 \#1750.

16. L. LeCam, Convergence in distribution of stochastic processes, Univ. Calif. Publ.

Statist. 2 (1957), 207-236. MR 19, 128.

17. E. J. McShane, A Riemann-type integral that includes Lebesgue-Stieltjes, Bochner and stochastic integrals, Mem. Amer. Math. Soc. No. 88 (1969). MR 42 \#36.

18. A. L. Peressini, Ordered topological vector spaces, Harper \& Row, New York and London, 1967. MR 37 \#3315. \#1689.

19. H. Schaefer, Topological vector spaces, Macmillan, New York, 1966. MR 33

20. F. D. Sentilles and D. C. Taylor, Factorization in Banach algebras and the general strict topology, Trans. Amer. Math. Soc. 142 (1969), 141-152. MR 40 \#703.

21. F. D. Sentilles, The strict topology on bounded sets, Pacific J. Math. 34 (1970), 529-540. MR 42 \#8283.

22. Conditions for equality of the Mackey and strict topologies, Bull. Amer. Math. Soc. 76 (1970), 107-112. MR 40 \#768.

23. - Bounded continuous functions on a completely regular space, Trans. Amer. Math. Soc. 168 (1972), 311-336. MR 45 \#4133.

24. V. Varadarajan, Measures on topological spaces, Mat. Sb. 55 (97) (1961), 35-100; English transl., Amer. Math. Soc. Transl. (2) 48 (1965), 161-228. MR 26 \#6342.

25. J. Wells, Bounded continuous vector-valued functions on a locally compact space, Michigan Math. J. 12 (1965), 119-126. MR 31 \#593.

26. A. Wiweger, Linear spaces with mixed topology, Studia Math. 20 (1961), 47-68. MR 24 \#A3490.

\section{DEPARTMENT OF MATHEMATICS, SOUTHERN ILLINOIS UNIVERSITY, CAR- BONDALE, ILLINOIS 62901}

Current address: Instituto de Mathemática, Universidade de Campinas, Caixa Postal 1170, 13100-Campinas, São Paulo, Brasil 